\title{
Experimental Analysis of the Fluidization Process of Binary Mixtures of Solids ${ }^{\dagger}$
}

\author{
B. Formisani and R. Girimonte \\ Dipartimento di Ingegneria Chimica e \\ dei Materiali, Università della Calabria*
}

\begin{abstract}
The simultaneous treatment of dissimilar solids is encountered in a number of processes that exploit fluidization technology (granulation, combustion, pyrolysis, etc.), but a satisfactory description of multicomponent fluidization dynamics is far from being achieved even for the relatively simple case of binary fluidization.

This paper discusses the fluidization properties of two-component beds of solids differing either in particle density or diameter. It is shown that although the minimum fluidization velocity of the mixture can be calculated by fully theoretical equations, it has very little meaning if referred to a twocomponent particle system. Experiments on both kinds of mixtures demonstrate that the binary fluidization process occurs within a characteristic velocity range whose boundaries coincide with the "initial" and the "final fluidization velocity" of the particle mixture. Substitution of the conventional concept of $u_{m f}$ by these parameters allows us to recognize that the fluidization dynamics of any binary mixture is determined by the initial arrangement of the fixed bed, as well as by the system composition and size or density ratio of its components.
\end{abstract}

\section{Introduction}

The fluidization dynamics of multicomponent particle beds has been the subject of a large number of experimental investigations in the last three decades. This persistent research effort is motivated by the technical and economic importance of the industrial processes which contemplate the simultaneous contact of a gaseous stream with two or more solids. These include fluidized bed combustors and gasifiers, waste incinerators, powder granulators, polymerization units and other types of reactors and contactors.

The variety of particle mixtures employed in these operations is fairly large. Based on the specific requirements of each application, the solid charge may be constituted by two or more types of materials. In turn, these may differ in one or more of their constitutive properties (particle size, density, shape, etc.), so that the mechanism through which a multi-

* I 87030 Arcavacata di Rende (Cosenza), Italy

${ }^{\dagger}$ Accepted: April, 2003 component particle bed enters into the fluidized state is not expected to be unique. Indeed, any given mixture exhibits a peculiar fluidization pattern whose characteristics are determined by the opposite tendencies of its components to mix or to segregate during suspension into the gaseous stream. Depending on the mixture properties as well as on the whole set of operating conditions, steady fluidization gives rise to a stable state of mixing of the solid components that may range from almost total segregation into distinct layers to practically complete mixing. In many practical situations, however, an intermediate component distribution is found, so that every component exhibits a particular concentration profile along the bed height. A major concern for these types of processes is therefore that of setting the operating conditions in a way that advantages associated with either mixing or segregation of the solid species can be exploited. The former regime is, for instance, the essential condition for ensuring uniform thermal properties and homogeneous product quality whenever required, whereas the latter can be exploited for conducting staged processes in distinct regions of the same bed.

At a lower degree of complexity, the equilibrium 
botwen mixing and segregation phenomena is also the distinctive feature of the fluidization pattern of W beds of only two solids. Because it should represent a relatively simple problem, investigating the fluidization behaviour of binary mixtures is considered an essential step towards the analysis of more complex systems. At the same time, two-component fluidization is normally considered a subject of practical interest on its own, due to its present uses in the area of coal processing, mineral solids handling, particle mixing and separation, etc. [1].

The intrinsic tendency of these systems to continuously change their component distribution while approaching the suspended state and the large number of factors that seem to influence the fluidization mechanism are the main sources of difficulty. Because of that, the currently available descriptions of two-solid fluidization are essentially empirical, whereas the extension of the basic principles of the fluidization theory to binary mixtures, as a preliminary condition for tackling more complex systems, has not yet been achieved. Based on experiments, this paper will discuss some points which are essential for analysing the behaviour of binary fluidized bed systems.

\section{Experimental}

All the experiments of this study were carried out in a transparent fluidization column of $10 \mathrm{~cm} \mathrm{ID,}$ equipped with a 4-mm-thick plastic porous distributor, ensuring high head loss and good gas distribution. The fluidizing gas was compressed air, whose flow rate was monitored in the range $0-25000 \mathrm{Nl} / \mathrm{h}$. Pressure drops across the whole particle bed were measured by a U-tube water manometer connected to a tap located $1 \mathrm{~mm}$ above the distributor plane, while three graduated scales spaced at $120^{\circ}$ around the column wall were used to determine the bed height and the bed voidage:

$$
\varepsilon_{0}=1-\frac{\mathrm{m} / \rho}{A H}
$$

Because all the solids used in the experiments belong to group B of Geldart's classification and show no homogeneous expansion when fluidized, the fixed bed voidage $\varepsilon_{0}$ was always considered equal to $\varepsilon_{m f}$.

Measurements were performed on three types of spherical solids: glass ballotini (GB), molecular sieves (MS), and steel shots (SS). The properties of each cut are listed in Table 1. The same table also reports the density and size ratios of the components of the four
Table 1 Properties of solids and mixtures

\begin{tabular}{|c|c|c|c|c|c|c|}
\hline Solid & $\begin{array}{c}\rho \\
{\left[\mathrm{g} / \mathrm{cm}^{3}\right]}\end{array}$ & $\begin{array}{c}\text { Sieve size } \\
{[\mu \mathrm{m}]}\end{array}$ & $\begin{array}{c}d \\
{[\mu \mathrm{m}]}\end{array}$ & Mixture & $\rho_{j} / \rho_{f}$ & $d_{j} / d_{f}$ \\
\hline GB154 & & $125-180$ & 154 & GB499-GB271 & 1 & 1.84 \\
GB271 & & $250-300$ & 271 & & & \\
\cline { 5 - 7 } GB428 & 2.48 & $400-500$ & 428 & GB612-GB154 & 1 & 3.97 \\
GB499 & & $350-600$ & 499 & & & \\
\cline { 5 - 7 } GB593 & & $500-710$ & 593 & SS439-GB428 & 3.06 & 1.03 \\
GB612 & & $600-710$ & 612 & & & \\
\hline MS624 & $1.46 *$ & $600-710$ & 624 & GB593-MS624 & 1.70 & 0.95 \\
\hline SS439 & 7.60 & $400-500$ & 439 & & & \\
\hline
\end{tabular}

mixtures used throughout the experimental campaign. Two of them (GB593-MS624 and SS439GB428), made of materials having approximately the same average diameter, are used to investigate the role of density difference on binary fluidization, whereas the other two (GB499-GB271 and GB612GB154) were used to elucidate the role of size difference.

The fluidization behaviour of each mixture was studied with reference to two extreme states of mixing of the fixed bed. Thus, well-mixed and fully segregated beds were the object of a distinct series of measurements.

In each of these series, a bed ratio $H / D$ close to 1.7 was maintained, so that the mixture concentration could vary by adjusting the solid concentration of each component.

\section{The essential features of binary fluidization}

From the experimental viewpoint, the minimum fluidization velocity of the bed is usually determined on a diagram $\Delta p$ versus $u$ similar to that of Fig. $\mathbf{1}$, at the intersection of the fixed bed curve with the horizontal line representing the suspended state.

When another solid of a different diameter is uniformly mixed, in any proportion, to the same material so as to form a binary mixture, the onset of fluidization throughout the bed becomes a gradual process. As sketched in Fig. 2a, at a certain velocity value a moving fluidization front establishes itself at the top of the bed. As the gas flow rate is increased, it moves across the bed down to the bottom of the column until, at a new characteristic velocity value, the whole bed is brought into a fully fluidized state. In the upper region of the bed, at its free surface, a bubbling layer builds up which contains almost exclusively one of 


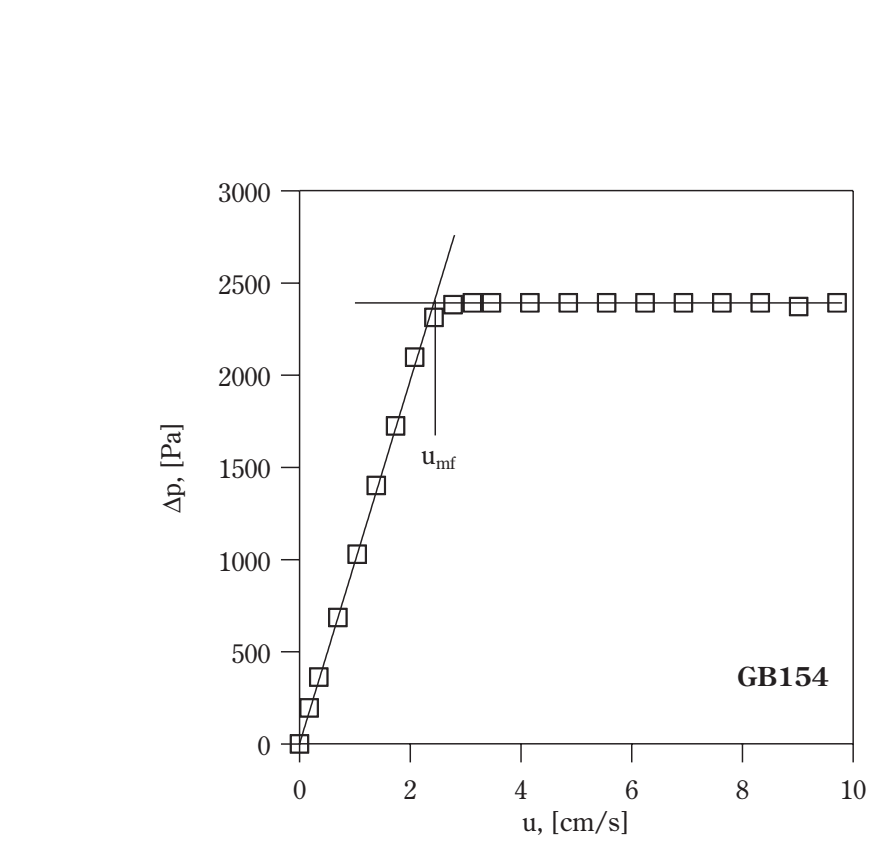

Fig. 1 Fluidization of a monodisperse particle bed.

the two species (the 'flotsam', according to the terminology introduced by Rowe et al. [2]) whilst the other solid (the 'jetsam' component) sinks and forms a defluidized layer just behind the fluidization front. The fluidization process for the mixture is thus accompanied by solids segregation up to a new velocity threshold at which the bed is fully fluidized (both solids mix again and bubbles flow freely).

The peculiarities of this mechanism are fully reflected by the experimental pressure drop diagram of Fig. 2b, where two characteristic velocity thresholds can be recognized $[3,4,5]$. They are the "initial fluidization velocity", $u_{i f}$, at which $\Delta p$ first deviates from the fixed bed curve, and the "final fluidization velocity", $u_{f f}$, at which the ultimate value of $\Delta p$ is first attained. Thus, these two limits identify the velocity range within which the entire particle collective undergoes suspension into the gaseous stream. Although it refers to a particular type of mixture (obtained by the complete mixing of spheres differing only in diameter), the diagram shows a common feature of any binary fluidization process, i.e. that their transition to the fluidized state is never instantaneous.

\section{Minimum fluidization velocity of a binary mixture}

The fluidization of binary beds is generally analysed by defining a "minimum fluidization velocity" $u_{m f}$ to be determined (as done with monosolid systems) at the intersection between the fixed bed $\Delta p$ curve and the horizontal line relevant to the sus-

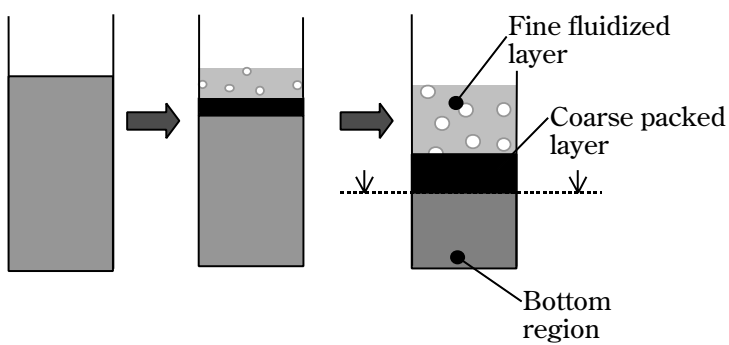

(a) Fluidization phenomenology

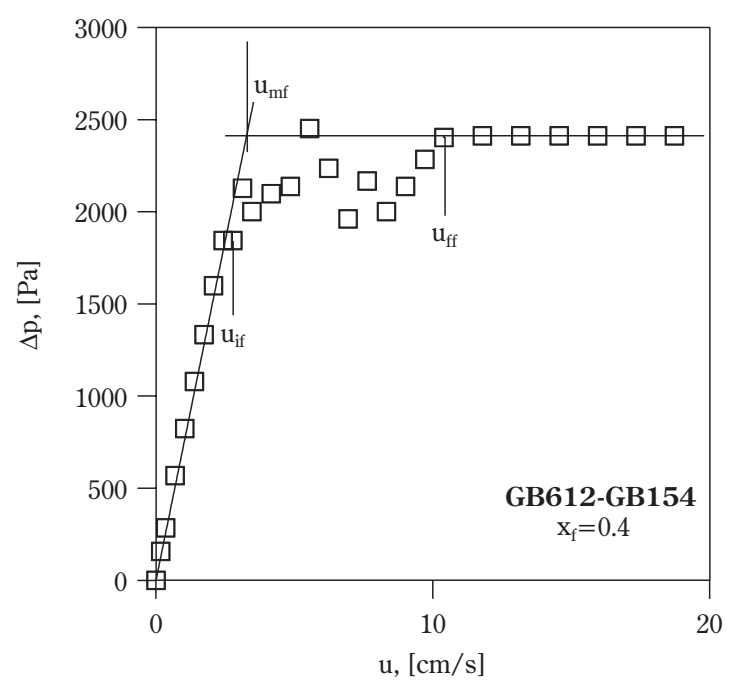

(b) Pressure drop diagram

Fig. 2 Fluidization of a two-component mixture.

pended state (see Fig. 2b).

It has often been reported that, given the mixture composition, $u_{m f}$ is not unique, as it can significantly change with the solids distribution within the fixed bed [6-9]. Moreover, different values of $u_{m f}$ are obtained depending on whether it is measured at increasing or decreasing gas velocity. Such effects are due to the peculiarity of the mixing/segregation pattern which accompanies the fluidization process. As discussed in a previous work on size segregating mixtures [10], this pattern is determined by the arrangement of the fixed bed.

Therefore, the variables that affect the mixing/segregation dynamics are also the ones upon which the minimum fluidization velocity of the binary bed is expected to depend. The trends of $u_{m f}$ as a function of the mixture composition (represented by the flotsam component volume fraction $x_{f}$ ) were analysed in two 
darferent cases pointing out the influence of the particlekdensity and the particle size distribution on the Wluidization pattern. This analysis was conducted on binary beds having opposite mixing states of their components, namely on perfectly mixed and thoroughly segregated systems.

For two-density beds, the results relevant to both homogeneous and segregated mixtures GB593MS624 and SS439-GB428 are reported in Fig. 3. For these types of systems, the dependence of $u_{m f}$ on $x_{f}$ is always linear, so that the equation

$$
u_{m f}=x_{f} u_{m f, f}+\left(1-x_{f}\right) u_{m f, j}
$$

proposed by Otero and Corella [11] applies. Irrespec-
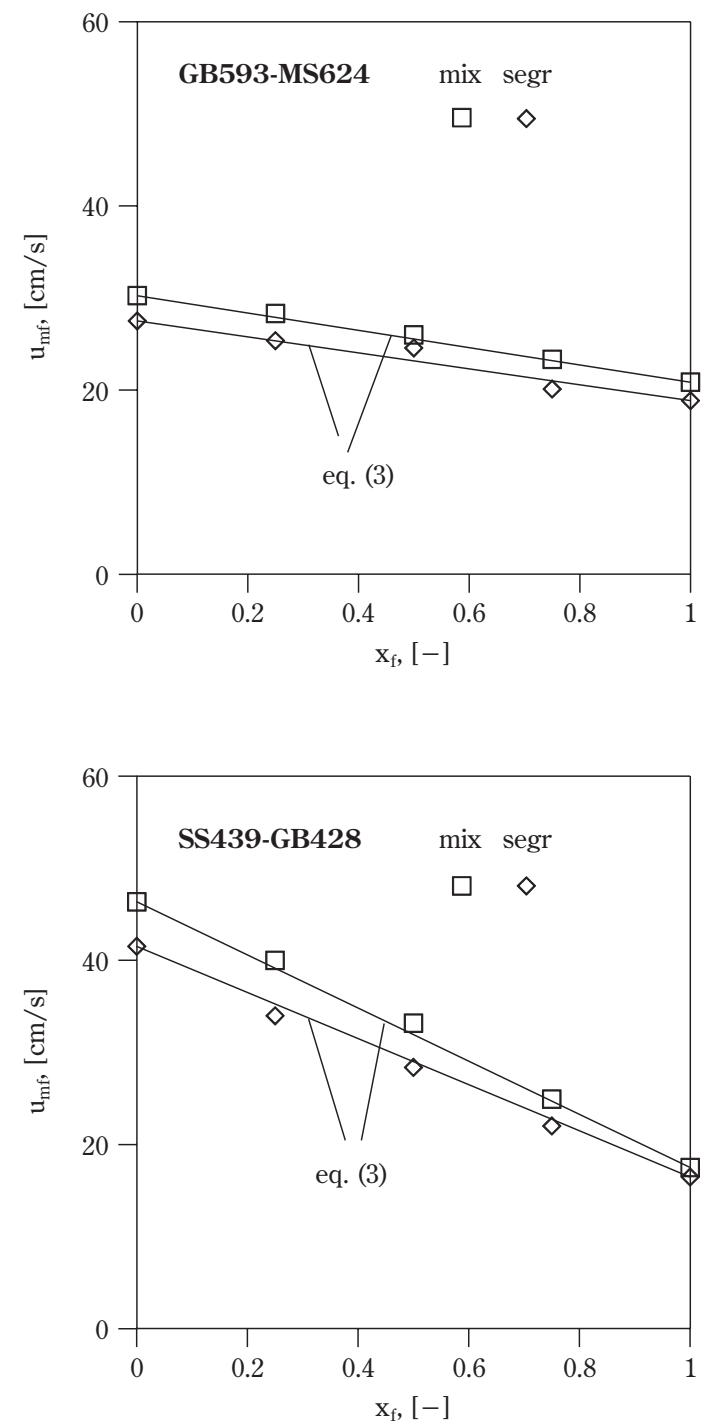

Fig. 3 Density segregating mixtures. Dependence of the minimum fluidization velocity on composition. tive of the component distribution, practically identical results are obtained with minor differences due to small variations in system voidage.

When, instead, mixture components are solids which differ only in diameter, as in the case of systems GB499-GB271 and GB612-GB154, the trends of $u_{m f}$ versus $x_{f}$ are quite different.

If the initial arrangement of the bed is such that the particles are well mixed, the relationship between the incipient fluidization threshold and the mixture composition is no longer linear. As observed also by other authors [12-14] and illustrated here in Fig. 4, experiments show a rapid fall of $u_{m f}$ at increasing flotsam fraction, followed by a more gradual decrease at
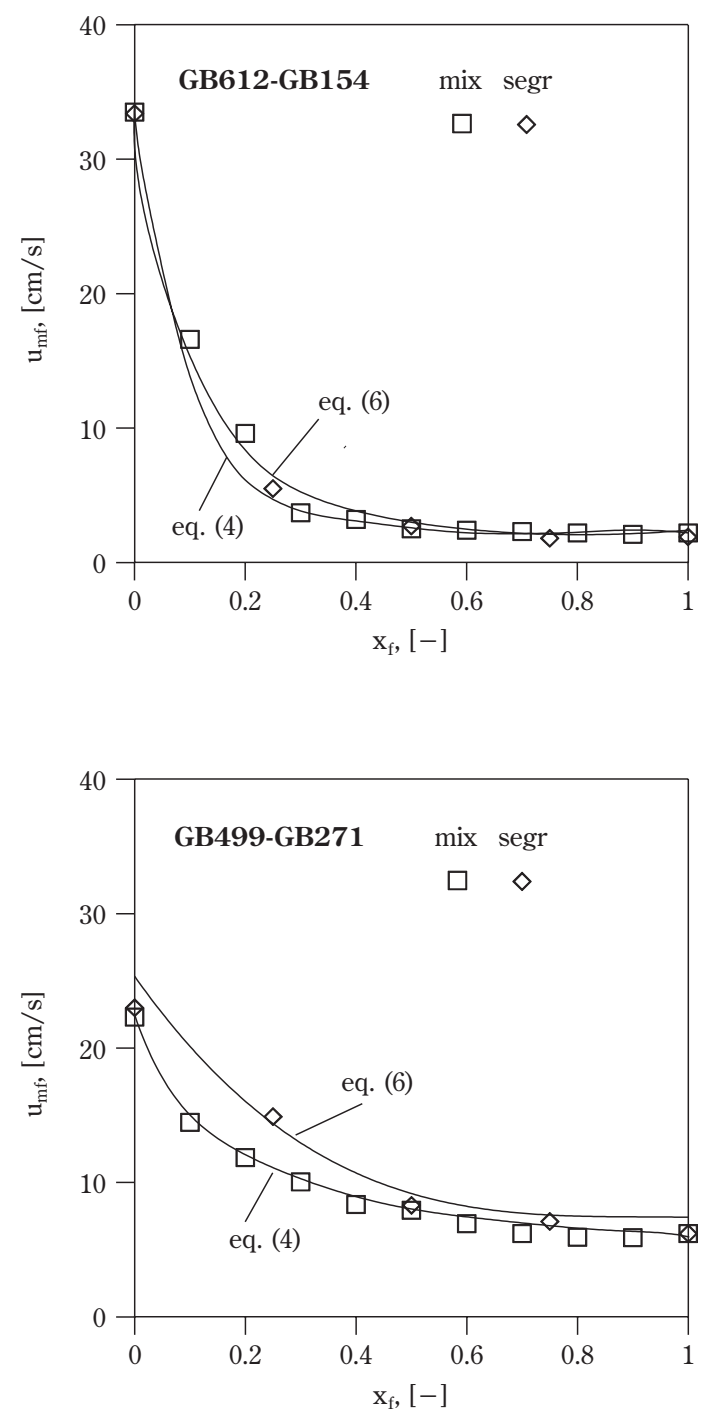

Fig. 4 Size segregating mixtures. Dependence of the minimum fluidization velocity on composition. 
higher values of $x_{f}$. Similar trends are shown also by fnditsan-on-jetsam segregated beds, with higher values of $u_{m f}$ at almost all $x_{f}$ s.

Altogether, these data demonstrate that density and size differences between the two solids promote distinct mechanisms of fluidization in the mixture, so that the dependence of $u_{m f}$ on either factor has to be analysed separately. In each case, a clear influence is exerted by the initial arrangement of the mixture: for a well-mixed bed, the transition to the fluidized state is associated with partial segregation of its components, while partial mixing is observed during the suspension process of an initially stratified particle system. Such almost opposite behaviour patterns cannot be represented by the conventional concept of minimum fluidization velocity, which does not capture their particular features.

\section{Interpretation}

A large number of empirical correlations, recently reviewed by $\mathrm{Wu}$ and Baeyens [15], have been proposed for calculating the minimum fluidization velocity of different kinds of binary mixtures. However, at least for the systems referred to in this paper, a fully theoretical analysis can be performed.

To this end, it must be remembered that when extended from monocomponent to binary beds of particles, the conventional definition of the minimum fluidization velocity provides a value of $u_{m f}$ which is determined by equating the pressure drop across the fixed bed and the buoyant weight per unit section of the solid mass.

When dealing with mixtures of particles which differ only in density, it may be assumed that the incipient fluidization voidage of the bed is practically unaffected by both component composition and mixing state. Thus, both for mixed and segregated systems, it is possible to rewrite the Carman-Kozeny equation as follows:

$$
180 \frac{\mu_{g} u_{m f}}{d^{2}} \frac{\left(1-\varepsilon_{m f}\right)^{2}}{\varepsilon_{m f}^{3}}=\left[\left(\rho_{f}-\rho_{g}\right) x_{f}+\left(\rho_{j}-\rho_{g}\right)\left(1-x_{f}\right)\right] g\left(1-\varepsilon_{m f}\right)(3)
$$

It is easy to verify that Equation 3 is equivalent to Equation 2 and fully independent of the initial distribution of the two solid species. This explains why, as shown in Fig. 3, it provides identical and good predictions of $u_{m f}$ both for homogeneous and segregated mixtures.

With regard to beds of different size distribution and initial mixing state, the bed voidage turns out to be strongly dependent on the composition [16] as well as on the particle diameter ratio $d_{j} / d_{f}$. For the mixtures indicated as GB499-GB271 and GB612GB154, experimental curves of $\varepsilon_{m f}$ versus $x_{f}$ are reported in Fig. 5.

As far as the $\varepsilon_{m f}$ variation is concerned, the equation:

$$
180 \frac{\mu_{g}}{d_{a v}^{2}} u_{m f, M} \frac{\left(1-\varepsilon_{m f, M}\right)^{2}}{\varepsilon_{m f, M}^{3}}=\left(\rho-\rho_{g}\right) g\left(1-\varepsilon_{m f, M}\right),
$$

with the surface/volume average particle diameter being given by the relationship:

$$
\frac{1}{d_{a v}}=\frac{x_{f}}{d_{f}}+\frac{1-x_{f}}{d_{j}}
$$

is capable, as shown in Fig. 4, to represent the experimental measurements.

When the same solids are initially arranged in the fixed bed as two segregated layers, with the fine component on top, the data of Fig. $\mathbf{4}$ are well represented by the equation proposed by Chiba et al. [6]

$$
\begin{aligned}
& 180 \mu_{g} u_{m f, S}\left[\frac{\left(1-\varepsilon_{m f, f}\right)^{2}}{\varepsilon_{m f, f}^{3}} \frac{H_{f}}{d_{f}^{2}}+\frac{\left(1-\varepsilon_{m f, j}\right)^{2}}{\varepsilon_{m f, j}^{3}} \frac{H_{j}}{d_{j}^{2}}\right] \\
& =\left(\rho-\rho_{g}\right) g\left[\left(1-\varepsilon_{m f, f}\right) H_{f}+\left(1-\varepsilon_{m f, j}\right) H_{j}\right],
\end{aligned}
$$

with

$$
x_{f}=\frac{H_{f}\left(1-\varepsilon_{m f, f}\right)}{H_{f}\left(1-\varepsilon_{m, f}\right)+H_{j}\left(1-\varepsilon_{m f, j}\right)}
$$

Equations 3, 4 and 6 make it possible to obtain, without introducing any adjustable parameter, good estimates of $u_{m f}$ for any mixture composition provided

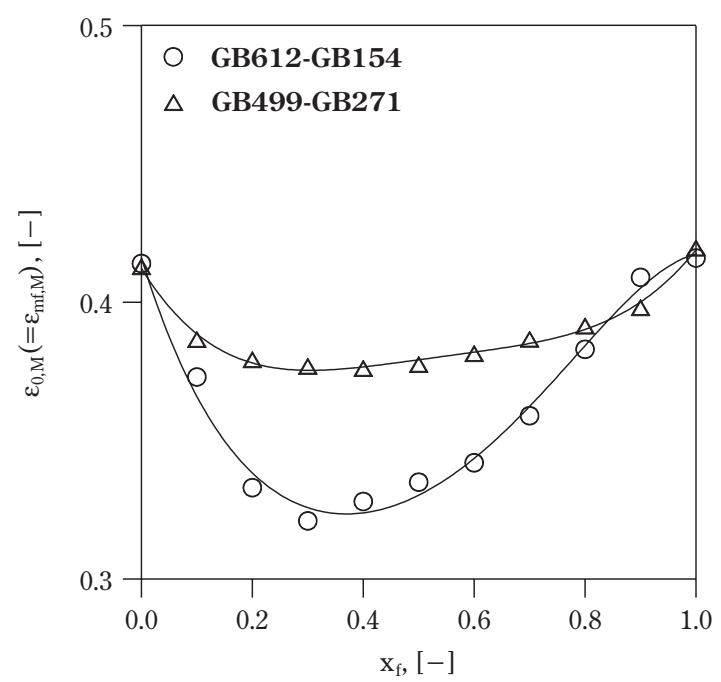

Fig. 5 Voidage of the well-mixed beds versus flotsam component average fraction. 
they are in one of the two extreme states of mixing represented by homogeneous and segregated beds.

\section{A clearer approach to binary fluidization}

Going by what prevails in the literature, devising predictive equations for $u_{m f}$ should be considered a key result for a deeper analysis of binary fluidization. Indeed, when addressing the mechanism of solids segregation, most authors have agreed that it is driven by bubbles flowing through the bed. This interpretation is based on observations similar to those reported earlier by Rowe et al. [17], who showed that flotsam particles are able to migrate upwards in the wake of bubbles, thus accumulating in the upper region of the bed. At the same time, the tendency of jetsam particles to sink to the bottom of the column has been generally interpreted to be an effect of the displacement caused by bubbles within the dense phase.

It is not quite clear whether the action of bubbles determines the segregation equilibrium or is just dynamically superimposed to it. It has induced many authors to look for a relationship between segregation and bubble flow rate, and several empirical equations have been proposed to relate the degree of mixing of the bed components to the excess gas velocity $u-u_{m f}$ $[14,15,18]$.

However, quantifying the intensity of bubbling by this parameter, as is usually done with monocomponent beds, assumes that both $u_{m f}$ and $u-u_{m f}$ keep their physical meaning even when applied to two-component systems. This implicit assumption turns out to be wrong. Reverting to Fig. $\mathbf{2 b}$, it can easily be observed that at $u_{m f}$, the upper region of the bed, which had begun to enter fluidization at $u_{i}$, is already bubbling, whereas the lower part of it is still packed. Even clearer is the case of segregated mixtures whose jetsam component initially forms the top layer. As shown in Fig. 6 for the case of GB612-GB154, the experimental curve of $\Delta p$, related to an unstable mechanism of fluidisation analysed in detail elsewhere [19], shows that the total pressure drop increases over the buoyant weight per unit section of the particle mass, so that the conventional definition of the minimum fluidization velocity corresponds to a fluid dynamic condition at which the whole bed is still in the fixed state. These results demonstrate that in binary fluidisation, particle suspension and bubbling are simultaneous rather than sequential phenomena and that the parameter $u_{m f}$ is not as significant as it is for monosolid beds. The excess gas velocity $u-u_{m f}$

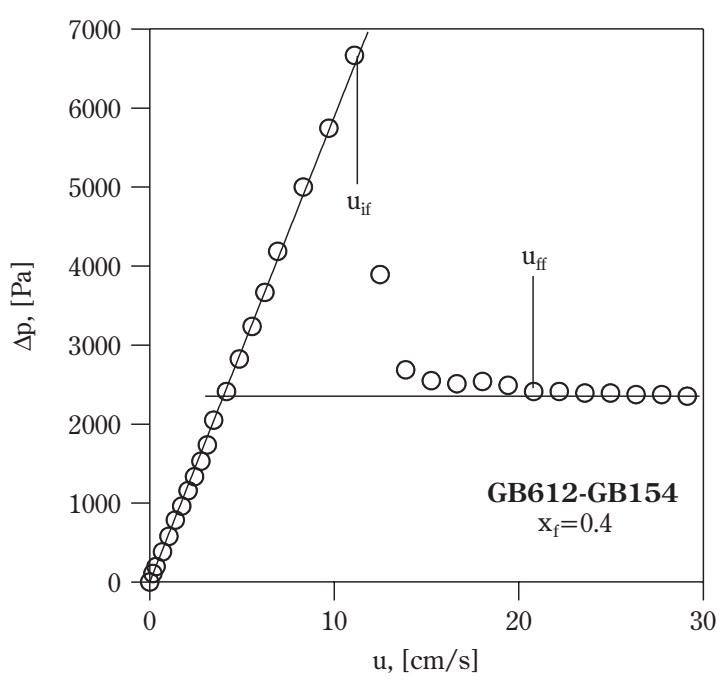

Fig. 6 Pressure drop diagram of a jetsam-on-flotsam segregated binary mixture.

can therefore by no means be used for measuring the intensity of the bubbling regime and the controlling factor of segregation.

Addressing the real phenomenology of binary fluidization systems can be done by developing an approach based on the definition of the "initial" and "final fluidization velocity". As mentioned in Section 3, these parameters constitute the lower and upper boundary of the interval of velocity along which any two-solid bed is crossed, from top to bottom, by the fluidization front.

Based on this, it is $u_{f f}$, rather than $u_{m f}$, that has to be viewed as the velocity at which the entire mixture attains the fluidized condition [3-5,9]. This velocity normally coincides with that of full mixing [10], while the difference between $u_{f f}$ and $u_{i f}$ is the amplitude of the velocity range within which mixing/segregation phenomena occur [20]. A systematic investigation of the dependence of both the "initial" and "final fluidization velocity" on variables which characterize binary mixtures is therefore of great importance for an accurate description of their fluidization properties. Two of these variables that surely play a major role, namely solids concentration and particle size distribution, have been the object of a series of experiments performed separately on density and size segregating systems.

For the former types of beds, represented by the mixtures GB593-MS624 and SS439-GB428, the trends of $u_{i f}$ and $u_{f f}$ at varying flotsam fraction $x_{f}$ are reported in Fig. 7 for the well-mixed arrangement, and in 

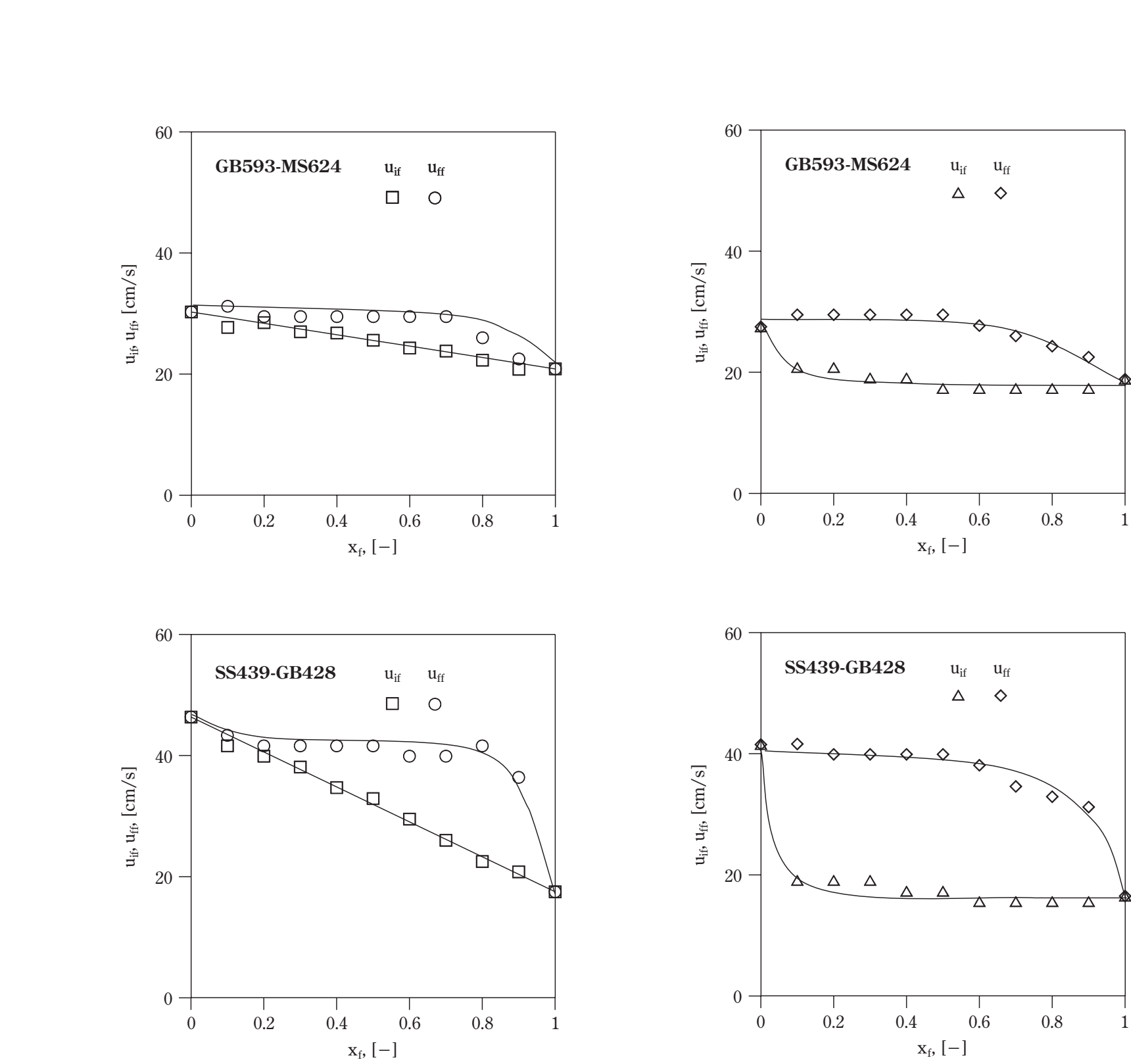

Fig. 7 Fluidization velocity range of density segregating mixtures. Well-mixed beds.

Fig. 8 for the initially segregated one.

The comparison between the results obtained for each mixture in the two cases clearly shows that the dependence of $u_{f f}$ on $x_{f}$ is practically unaffected by the initial arrangement of the bed. For a wide range of compositions, its values are close to that of the minimum fluidization velocity of the jetsam component, with a visible decrease at high flotsam concentration. With regard to the dependence of $u_{i f}$ on $x_{f}$, when the initial mixture is homogeneously distributed (Fig. 7), the initial fluidization velocity of the bed is practically coincident with its $u_{m f}$ (i.e. with the weighed average of the minimum fluidization velocities of the two solids). When the initial bed is segregated (Fig. 8),

Fig. 8 Fluidization velocity range of density segregating mix-

tures. Segregated beds.

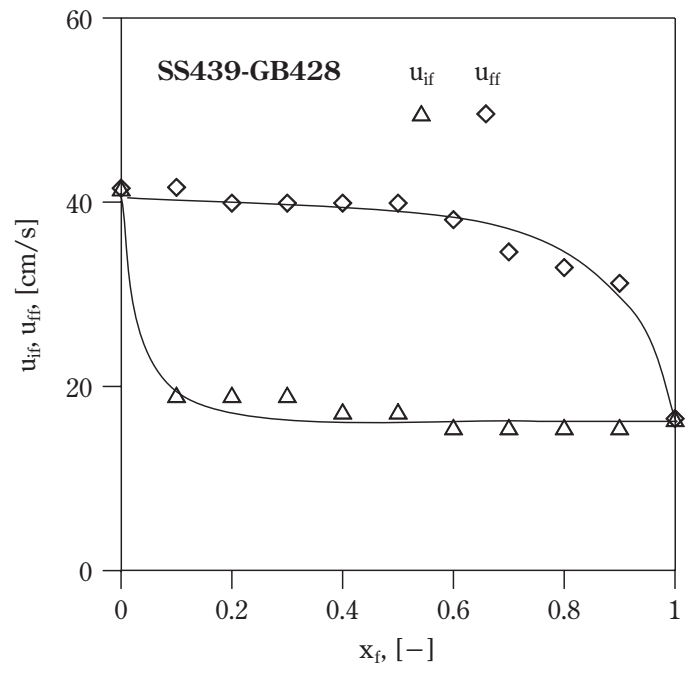

fluidization starts in the upper bed layer where the flotsam components are, so that $u_{i f}$ is always practically equal to the minimum fluidization velocity of the flotsam. Such results demonstrate also that the value of the solid density ratio $\rho_{j} / \rho_{f}$ does not alter the general dynamics of fluidization, although it determines the absolute amplitude of the velocity interval along which it takes place. Most important is that the analysis based on the two characteristic velocities shows that, for any density of segregating mixture, the fluidization pattern depends on the initial configuration of the fixed bed (Fig. 3).

The same analysis was performed on size segregating systems by examining the results reported in 
Fogs 9 and 10 for mixed and segregated beds, respectively.

As previously reported for two different density systems, the curve of $u_{f f}$ is not related to the initial arrangement of the mixture components. On the contrary, for both GB499-GB271 and GB612-GB154, the initial fluidization velocity of the mixed bed is, particularly at low flotsam fractions, higher than that of the stratified one. Fluidization starts at the free surface of the bed so that when its upper layer is fluidized, the rapid fall of $u_{i f}$ observed in both diagrams of Fig. 9 is mainly due to the voidage reduction accompanying the increase of the flotsam fraction. Beyond the value of $x_{f}$ that corresponds to the minimum of each curve in Fig. 5, the mixture voidage increases again, but
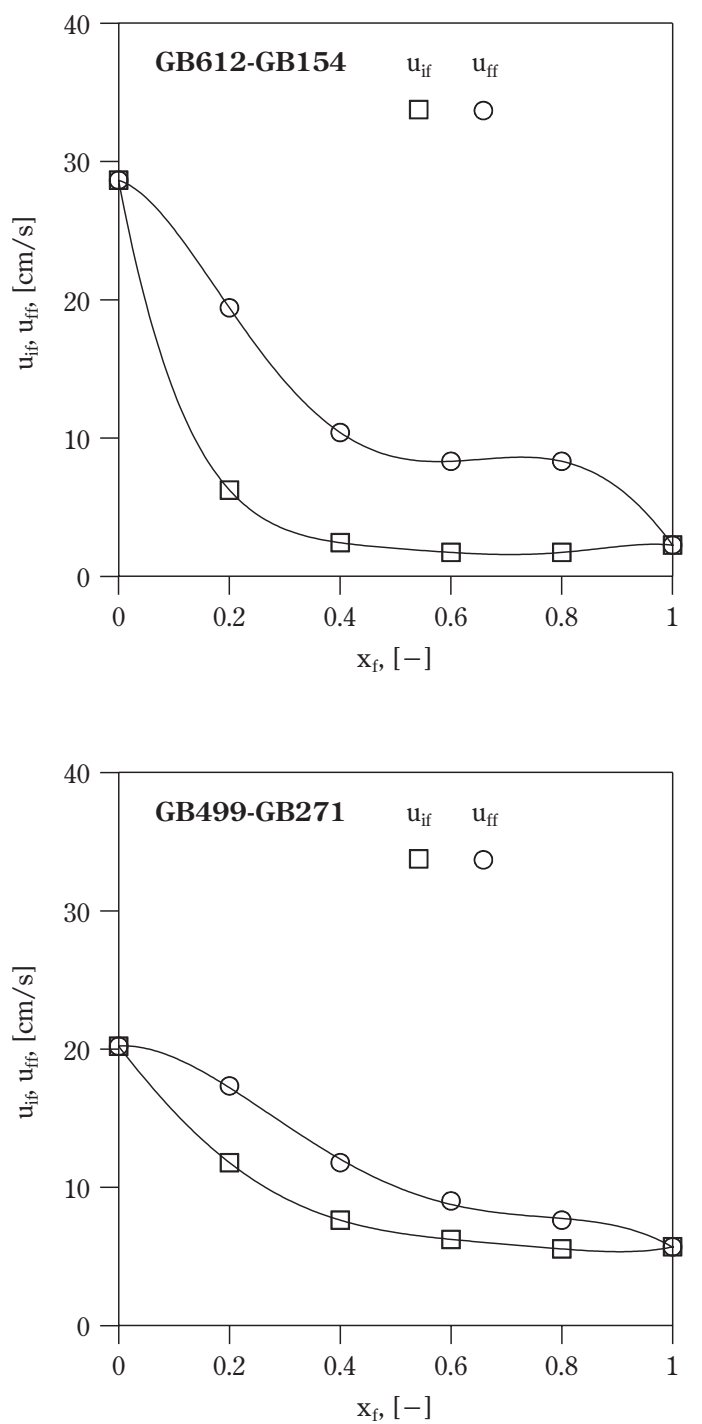

Fig. 9 Fluidization velocity range of size segregating mixtures. Well-mixed beds. the total weight of the particle mass per unit section keeps on decreasing, so that $u_{i f}$ approaches its ultimate value (i.e. $u_{m f, f}$, at $x_{f}=1$ ) more gradually. In contrast, for the segregated systems, $u_{i f}$ coincides, at practically all $x_{f} \mathrm{~s}$, with the minimum fluidization velocity of the flotsam component, below which the jetsam layer acts as a passive gas distributor. That explains why the values of $u_{i f}$ in Fig. 10 are practically unaffected by the mixture composition.

Given these trends of the characteristic velocities, the amplitude of the fluidization field is found to depend on the mixture composition as well as on the initial state of mixing of the two solids. With regard to the component size ratio $d_{j} / d_{f}$, the influence of this parameter on $\left(u_{f f}-u_{i f}\right)$ is stronger than that exerted
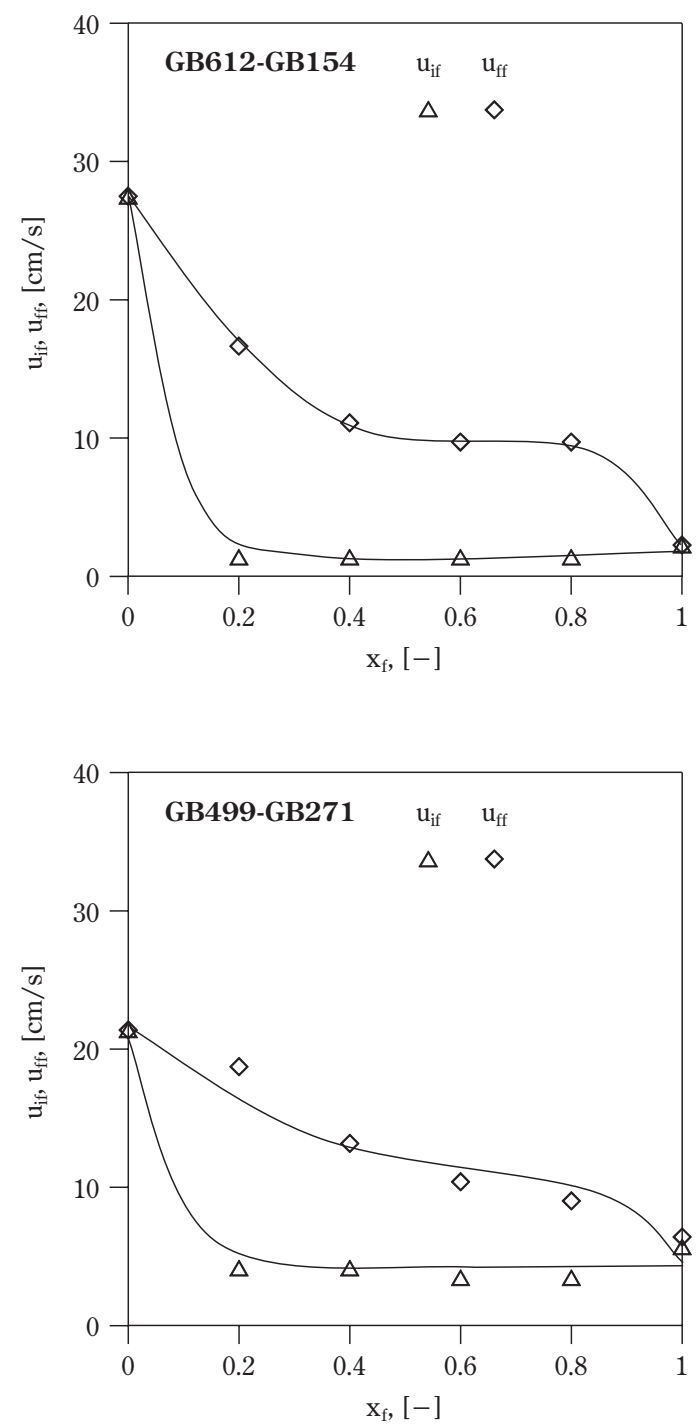

Fig. 10 Fluidization velocity range of size segregating mixtures. Segregated beds. 
on it by $\beta_{x} \rho_{f}$. Indeed, as already shown in Fig. 5, as long as the two solids are not fully segregated, the size ratio determines the value of the bed voidage.

The strong relationship between $d_{j} / d_{f}$ and $\varepsilon_{m f}$ at any mixture composition is therefore the main element of difference between the mechanisms of size and density segregating fluidization.

\section{Conclusions}

The fluidization of a two-solid mixture is a gradual process regulated by mixing/segregation phenomena that simultaneously change the component distribution of the bed.

The definition of a minimum fluidization velocity of the binary system, widely used in most literature studies, can result in a misleading analysis of its fluidization behaviour: although it can be calculated by rewriting theoretical equations such as CarmanKozeny's in a form suitable for taking the specific nature of each mixture into account, $u_{m f}$ does not represent the actual fluidization condition. As a consequence, it also constitutes a source of error for models which relate segregation to the bubble flow rate measured by the excess gas velocity $u-u_{m f}$.

It is possible to develop a clearer approach based on definition of the "initial" and "final fluidization velocity" of the binary mixture and closer to their actual phenomenology of fluidization. These parameters are the lower and upper boundary, respectively, of a characteristic fluidization velocity range whose amplitude, measured by the difference between $u_{f f}$ and $u_{i f}$, is mainly determined by the density or size ratio between the two components as well as by the mixture composition and component distribution within the fixed bed, whose influence is clearly highlighted.

\section{Acknowledgements}

This work was partially supported by the Italian Ministry of University and Research (MIUR), within the scope of the "PRIN 2001" program.

The authors are grateful to Mr R. Mazzitelli for his careful work in sample preparation and granulometric analysis.

\section{List of symbols}

A column cross-section

[cm]

D column diameter

$d$ particle diameter $d_{a v} \quad$ average particle diameter

$[\mu \mathrm{m}]$

$g$ gravity acceleration

$H$ height of the bed, of the layer

$\left[\mathrm{cm} / \mathrm{s}^{2}\right]$

$m$ solids mass

$\Delta p \quad$ pressure drop

$[\mathrm{cm}]$

$u \quad$ superficial gas velocity $\quad[\mathrm{cm} / \mathrm{s}]$

$u_{i f}, u_{f f}$ initial, final fluidization velocity $\quad[\mathrm{cm} / \mathrm{s}]$

$u_{m f}$ minimum fluidization velocity $\quad[\mathrm{cm} / \mathrm{s}]$

$x_{f} \quad$ volume fraction of the flotsam component [-]

$\varepsilon_{0} \quad$ fixed bed voidage [-]

$\varepsilon_{m f} \quad$ minimum fluidization voidage

$\mu_{g} \quad$ gas viscosity

$[-]$

$\rho \quad$ solids density $\quad\left[\mathrm{g} / \mathrm{cm}^{3}\right]$

$\rho_{g} \quad$ gas density $\quad\left[\mathrm{g} / \mathrm{cm}^{3}\right]$

\section{Subscripts}

$f, j \quad$ of the flotsam, jetsam component

$M, S$ of the well-mixed, segregated bed

\section{References}

1) Nienow, A. W. and Chiba, T.: "Fluidization of dissimilar solids”, in J. F. Davidson, D. Harrison, and R. Clift (Eds.), Fluidization, Academic Press, London, England, pp.357-382 (1985)

2) Rowe, P. N., Nienow, A. W. and Agbim, A. J. (1972). The mechanism by which particles segregate in gas fluidised beds-binary systems of near-spherical particles. Transactions of the Institution of Chemical Engineers, 50, 310-323.

3) Chen, J. L.-P. and Keairns, D. L. (1975). Particle segregation in a fluidized bed. The Canadian Journal of Chemical Engineering, 53, 395-402.

4) Vaid, R. P. and Sen Gupta P. (1978). Minimum fluidization velocities in beds of mixed solids. The Canadian Journal of Chemical Engineering, 56, 292-296.

5) Carsky, M., Pata, J., Vesely, V. and Hartman, M. (1987). Binary system fluidized bed equilibrium. Powder Technology, 51, 237-242.

6) Chiba, S., Chiba, T., Nienow, A. W. and Kobayashi, H. (1979). The minimum fluidisation velocity, bed expansion and pressure-drop profile of binary particle mixtures. Powder Technology, 22, 255-269.

7) Yang, W.-C. and Keairns, D. L. (1982). Rate of particle separation in a gas fluidized bed. Ind. Eng. Chem. Fundam., 21, 228-235.

8) Thonglimp, V., Hiquily, N. and Laguerie, C. (1984). Vitesse minimale de fluidisation et expansion des couches de mélanges de particules solides fluidisées par un gaz. Powder Technology, 39, 223-239.

9) Noda, K., Uchida, S., Makino, T. and Kamo, H. (1986). Minimum fluidization velocity of binary mixtures of particles with large size ratio. Powder Technology, 46, 149154.

10) Formisani, B., De Cristofaro, G. and Girimonte, R. (2001). A fundamental approach to the phenomenology 
of aluidization of size segregating binary mixtures of

N.Solids. Chemical Engineering Science, 56, 1-11.

$\mathrm{P}^{\circ}$ N.11) Otero, A. R. and Corella, J. (1971). Anales de la RSEFQ $67,1207-1219$.

12) Formisani, B. (1991). Packing and fluidization properties of binary mixtures of spherical particles. Powder Technology, 66, 259-264.

13) Nienow, A. W., Rowe, P. N. and Cheung, L. Y.-L. (1978). A quantitative analysis of the mixing of two segregating powders of different density in a gas-fluidised bed. Powder Technology, 20, 89-97.

14) Rice, R. W. and Brainovich, J. F. (1986). Mixing/segregation in two- and three-dimensional fluidized beds: binary systems of equidensity spherical particles. A.I.Ch.E. Journal, 32, 7-16.

15) Wu, S. Y. and Baeyens, J. (1998). Segregation by size difference in gas fluidized beds. Powder Technology, 98, 139-150.

16) Yu, A. B. and Standish, N. (1987). Porosity calculations of multi-component mixtures of spherical particles. Pow- der Technology, 52, 233-241.

17) Rowe, P. N., Nienow, A. W. and Agbim, A. J. (1972). The mechanisms by which particles segregate in gas fluidised beds - binary systems of near-spherical particles. Transactions of the Institution of Chemical Engineers, 50, 310-323.

18) Nienow, A. W., Rowe, P. N. and Cheung, L. Y.-L. (1978) A quantitative analysis of the mixing of two segregated powders of different density in a gas fluidized bed. Powder Technology, 20, 89-97.

19) Formisani, B., Girimonte, R. and Guzzo, A. (2002). Unstable fluidization of size segregated binary mixtures with the coarser component initially on top. Proc. " 4 th World Congress on Particle Technology", Sydney, Australia.

20) Marzocchella, A., Salatino, P., Di Pastena, V. and Lirer, L. (2000). Transient fluidization and segregation by size difference of binary mixtures of particles. A.I.Ch.E. Journal, 46, 2175-2182.

\section{Author's short biography}
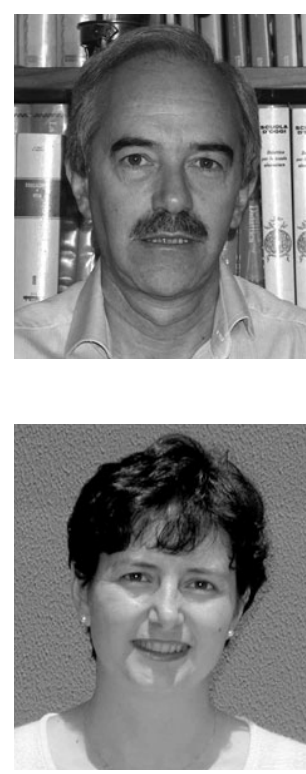

\section{Brunello Formisani}

Brunello Formisani graduated in Chemical Engineering from the University of Naples, Italy, in 1979. In 1984, he joined the Department of Chemical and Materials Engineering at the University of Calabria, where he has been an associate professor since 1992. His teaching activity involves chemical plant design and solids processing. His research interests are in the field of particle fluidization, and include packed-fluidized beds, fluidization of solid mixtures, high-temperature fluidization, and interparticle forces.

\section{Rossella Girimonte}

Rossella Girimonte graduated in Chemical Engineering from the University of Calabria, Italy, in 1995 and received her Ph.D. in 1999. She is presently an assistant professor in the Department of Chemical and Materials Engineering at the same university, where she holds a course on unit operations. Her research activity is currently focused on high-temperature fluidization and fluidization of multicomponent particle beds. 